\title{
MORE GENERALISED SYLOW THEOREMS
}

\section{J. HAWTHORN}

(Received 10 February 1995; revised 19 June 1995)

Communicated by R. Howlett

\begin{abstract}
The study of classes of finite groups is divided into two parts. The projective theory studies formations and Schunck classes. The dual injective theory studies Fitting classes. In each type of class a generalisation of Sylow's theorem holds. In this paper we seek further generalisations of Sylow's theorem which hold for classes which are neither injective nor projective, but obey other related properties. Firstly a common framework for the injective and projective theories is constructed. Within the context of this common framework further types of Sylow theorem can then be sought. An example is given of a property which is a simple hybrid of injectivity and projectivity which we will call 'interjectivity'. A generalised Sylow theorem is then proved in the interjective case.
\end{abstract}

1991 Mathematics subject classification (Amer. Math. Soc.): primary 20D10; secondary 20F17.

\section{Introduction}

The study of classes of finite groups is divided into two parts. The projective theory studies formations and Schunck classes. The dual injective theory studies Fitting classes. In each type of class a generalisation of Sylow's theorem holds, specifying that every solvable group contains, uniquely up to conjugacy, a subgroup which is maximal belonging to the class obeying certain conditions.

In the projective theory, this group is called a $\mathscr{K}$-projector, where $\mathscr{K}$ is the class of groups. A $\mathscr{K}$-projector $S$ of $G$ has the property that, for all $N \unlhd G$, the group $S N / N$ is a $\mathscr{K}$-maximal subgroup of $G / N$. The classes where every solvable group has a $\mathscr{K}$ projector (unique up to conjugacy), are called Schunck classes, and are characterised internally by two properties: Firstly they are closed under epimorphism; and secondly, if every primitive quotient of $G$ belongs to $\mathscr{K}$, then $G$ must also belong to $\mathscr{K}$.

In the injective theory, the group is called a $\mathscr{K}$-injector, and has the property that for

(C) 1997 Australian Mathematical Society 0263-6115/97 \$A2.00+0.00 
every $N \unlhd G, N \cap S$ is a $\mathscr{K}$-maximal subgroup of $N$. Classes where every solvable group has a $\mathscr{K}$-injector (unique up to conjugacy), are called Fitting classes, and are similarly characterised by two internal properties: Firstly closure under passing to normal (hence subnormal) subgroup; and secondly closure under normal product.

It is natural to ask whether these two flavours are the only ones possible, or whether other similar generalisations of Sylow's theorem exist. Before we can begin such a search, we must set up a general theory of what we shall call 'ectors, for which injectors and projectors are simply special cases. In other words we must build a common framework for the two theories that we have, before we can commence a search for other theories that might fit into such a framework.

The main problem that we face is illustrated by the example of the class $\mathscr{N}$ of nilpotent groups, which is both Schunck and Fitting. Hence $\mathscr{N}$-injectors and $\mathscr{N}$ projectors exist in any finite solvable group. In $S_{3}$ for example, the $\mathscr{N}$-injector is the Sylow 3-subgroup, while the $\mathscr{N}$-projector is the Sylow 2-subgroup. This creates a problem of ambiguity when we seek to create a theory of 'ectors where injectors and projectors are 'ectors with special properties. We will have to reformulate both existing theories to surmount this difficulty.

For further background reading on Schunck and Fitting classes, see [1], which has an excellent list of references.

\section{Subgroup classes}

DEFINITION 1. Let $\mathscr{X}$ be a class of finite groups. We define a subgroup class $\mathscr{C}$ of $\mathscr{X}$ to be a collection of pairs $(G, H)$ of groups with $H \leq G \in \mathscr{X}$, which is closed under isomorphism of pairs of groups, and which contains all pairs of the form $(G, G)$ where $G \in \mathscr{X}$.

This of course is a uselessly general definition. Before we can obtain any results we must endow our creation with more structure.

DEFINITION 2. A subgroup class $\mathscr{C}$ of $\mathscr{X}$ is said to be

(a) bounded, if $H \leq K \leq G,(G, H) \in \mathscr{C}$ implies $(G, K) \in \mathscr{C}$.

(b) closed, if $H \leq K \leq G,(G, H) \in \mathscr{C}$ and $K \in \mathscr{X}$ implies $(K, H) \in \mathscr{C}$.

(c) transitive, if whenever $H \leq K \leq G$ with $(G, K) \in \mathscr{C}$ and $(K, H) \in \mathscr{C}$ then $(G, H) \in \mathscr{C}$.

(d) complete, if it is closed, bounded, and transitive.

(e) conjective if $(G, H) \in \mathscr{C}$ and $(G, K) \in \mathscr{C}$ implies that there exists some $S \leq G$ with $(G, S) \in \mathscr{C}$ and $S \leq H^{h}, S \leq K^{k}$ for some $h, k \in G$. In the case when $\mathscr{C}$ is bounded, this simplifies to the condition that whenever $(G, H) \in \mathscr{C}$ and $(G, K) \in \mathscr{C}$ then $\left(G, H \cap K^{g}\right) \in \mathscr{C}$ for some $g \in G$. 
Note that the role of $\mathscr{X}$ in this paper is merely to define the universe of groups with which we are working. Later we will restrict ourselves to the case that $\mathscr{X}$ is the class of all solvable groups, which is the setting for the main results of this paper.

If $\mathscr{C}$ is conjective, then there exists in every group $G \in \mathscr{X}$, a subgroup $S_{G}$ which is minimal with $\left(G, S_{G}\right) \in \mathscr{C}$, and this subgroup is unique up to conjugacy. We call $S_{G}$ a $\mathscr{C}$-conjector of $G$.

If $\mathscr{X}$ is the class of solvable groups, and $\mathscr{K}$ is a Schunck class, then we can define a subgroup class $\mathscr{P}(\mathscr{K})$ of $\mathscr{X}$ by $(G, H) \in \mathscr{P}(\mathscr{K})$ if and only if $G \in \mathscr{X}$ and $H$ contains a $\mathscr{K}$-projector. It is easy to check that $\mathscr{P}(\mathscr{K})$ is complete and conjective, and $\mathscr{P}(\mathscr{K})$-conjectors are $\mathscr{K}$-projectors.

If $\mathscr{X}$ is the class of solvable groups, and $\mathscr{K}$ is a Fitting class, then we define a subgroup class $\mathscr{I}(\mathscr{K})$ of $\mathscr{X}$ by $(G, H) \in \mathscr{I}(\mathscr{K})$ if and only if $G \in \mathscr{X}$ and $H$ contains a $\mathscr{K}$-injector. It is easy to check that $\mathscr{I}(\mathscr{K})$ is complete and conjective, and $\mathscr{I}(\mathscr{K})$-conjectors are $\mathscr{K}$-injectors.

We note that $\mathscr{P}(\mathscr{N}) \neq \mathscr{I}(\mathscr{N})$. Thus the language of subgroup classes enables us to avoid the problem of ambiguity we noted earlier.

We now seek to characterise injectors and projectors purely in terms of the language of subgroup classes.

DEFINITION 3. A subgroup class $\mathscr{C}$ of $\mathscr{X}$ is said to be projective if whenever $K \leq H \leq G \in \mathscr{X}$ and $K \unlhd G$ with $G / K \in \mathscr{X}$, then $(G, H) \in \mathscr{C}$ if and only if $(G / K, H / K) \in \mathscr{C}$.

DEFINITION 4. A subgroup class $\mathscr{C}$ of $\mathscr{X}$ is said to be injective if it satisfies the following two conditions.

(a) If $N \unlhd G,(G, H) \in \mathscr{C}$ and $N \in \mathscr{X}$, then $(N, N \cap H) \in \mathscr{C}$.

(b) If $N \unlhd G \in \mathscr{X}$ with $(N, K) \in \mathscr{C}$ then there exists $S \leq G$ with $(G, S) \in \mathscr{C}$ and $S \cap N \leq K$.

Note that, if $\mathscr{K}$ is Schunck, then $\mathscr{P}(\mathscr{K})$ is projective, and if $\mathscr{K}$ is Fitting, then $\mathscr{I}(\mathscr{K})$ is injective.

We are now in a position to prove two theorems.

THEOREM 5. Let $\mathscr{X}$ be the class of solvable groups, and let $\mathscr{C}$ be a complete projective subgroup class of $\mathscr{X}$. Then $\mathscr{C}$ is also conjective.

PROOF. Let $G \geq H, K$ represent a counterexample to conjectivity which is minimal with respect to the order of $G$ and the indices of $H$ and $K$. Hence we are assuming that $(G, H) \in \mathscr{C},(G, K) \in \mathscr{C}$, and that $\left(G, H \cap K^{8}\right) \notin \mathscr{C}$ for all $g \in G$. We note that trivially neither $H$ nor $K$ can be equal to $G$. 
We now claim that $H$ and $K$ are both maximal subgroups of $G$. Indeed, assume without loss of generality that $H<T<G$. Then by boundedness, $(G, T) \in \mathscr{C}$. Hence $G \geq T, K$ obeys the conjectivity condition by the inductive assumption. Thus $\left(G, K^{g} \cap T\right) \in \mathscr{C}$ for some $g \in G$. But now, by closure, $(T, H) \in \mathscr{C}$ and $\left(T, K^{g} \cap T\right) \in \mathscr{C}$. Also $T \geq H, K^{g} \cap T$ obeys the conjectivity condition and thus $\left(T,\left(K^{g} \cap T\right)^{t} \cap H\right) \in \mathscr{C}$ for some $t \in T$. Thus $\left(T, K^{g t} \cap H\right) \in \mathscr{C}$ and also $(G, T) \in \mathscr{C}$, hence transitivity gives $\left(G, K^{g t} \cap H\right) \in \mathscr{C}$ which contradicts our assumption that $G \geq H, K$ does not obey the conjectivity condition. So $H$ and $K$ must indeed be maximal subgroups of $G$ as claimed.

Now let $M$ be an arbitrary minimal normal subgroup of $G$. Form the homomorphism with kernel $M$, and denote images under this homomorphism with a bar. Suppose $M \leq H \cap K$. Note that $(\bar{G}, \bar{H}) \in \mathscr{C}$ and $(\bar{G}, \bar{K}) \in \mathscr{C}$ by projectivity. Hence, by our inductive assumption, $\bar{G} \geq \bar{H}, \bar{K}$ obeys the conjectivity condition. Thus $\left(\bar{G}, \bar{H} \cap \bar{K}^{\bar{g}}\right) \in \mathscr{C}$ for some $\bar{g} \in \bar{G}$. But projectivity now gives $\left(G, H \cap K^{g}\right) \in \mathscr{C}$ which is a contradiction. Thus $M$ cannot be contained in both $H$ and $K$. Suppose $M$ is contained in exactly one of the groups $H$ and $K$. Assume without loss of generality that $M \leq H$, so $M \notin K, K M=G$ and $H=(H \cap K) M$. Now $\bar{G}=\bar{K}$ and $\overline{H \cap K}=\bar{H}$. By projectivity $(\bar{G}, \bar{H}) \in \mathscr{C}$. Hence $(\bar{K}, \overline{H \cap K}) \in \mathscr{C}$, and by projectivity we conclude that $(K, K \cap H) \in \mathscr{C}$. But transitivity now implies that $(G, K \cap H) \in \mathscr{C}$ which contradicts the inductive assumption. So $M$ cannot be contained in exactly one of the groups $H$ and $K$. We must conclude that $M$ is not contained in either $K$ or $H$.

We have thus shown that the groups $H$ and $K$ are maximal subgroups of $G$ containing no normal subgroups. Hence both are stabilisers of $G$, which must be a primitive group. But stabilisers are unique up to conjugacy (see [1] Theorem 15.6). So $H$ and $K$ are conjugate in $G$ and thus they trivially obey the conjectivity condition. There is no escape from this final contradiction.

Our second theorem deals with the injective case.

THEOREM 6. Let $\mathscr{X}$ be the class of solvable groups, and let $\mathscr{C}$ be a complete injective subgroup class of $\mathscr{X}$. Then $\mathscr{C}$ is also conjective.

PROOF. The proof begins in a similar fashion to the last proof by assuming that $G \geq H, K$ represents a counterexample to conjectivity which is minimal with respect to the orders of $G, H$, and $K$ respectively (cf. the indices of $H$ and $K$ last time).

Suppose that $(H, S) \in \mathscr{C}$ for some $S<H$. Then induction on $G \geq S, K$ gives $\left(G, S \cap K^{g}\right) \in \mathscr{C}$ for some $g \in G$. But $S \cap K^{g} \leq H \cap K^{g}$ implies that $\left(G, H \cap K^{g}\right) \in \mathscr{C}$, a contradiction. We conclude that there are no proper subgroups $S<H$ (respectively $S<K$ ) with $(H, S) \in \mathscr{C}$ (respectively $(K, S) \in \mathscr{C}$ ). 
Suppose that $H \leq M$ for some proper normal subgroup $M$ of $G$. Then $(G, M) \in \mathscr{C}$ and $(M, H) \in \mathscr{C}$ by completeness, and also $(M, M \cap K) \in \mathscr{C}$ by injectivity. Induction on the groups $M \geq H, M \cap K$ implies that $\left(M, M \cap K^{m} \cap H\right) \in \mathscr{C}$ for some $m \in M$. But transitivity now gives $\left(G, K^{m} \cap H\right) \in \mathscr{C}$, a contradiction. We conclude that neither $H$ nor $K$ is contained in a proper normal subgroup of $G$.

Assume that there exists a proper subgroup $X<G$ with $H \leq X$ and $K \leq X$. Then $(G, X) \in \mathscr{C}$, and also $(X, H) \in \mathscr{C}$ and $(X, K) \in \mathscr{C}$. Induction on $X \geq H, K$ implies $\left(X, H \cap K^{x}\right) \in \mathscr{C}$ for some $x \in X$. Transitivity gives $\left(G, H \cap K^{x}\right) \in \mathscr{C}$, a contradiction. Since we could have applied this argument to any conjugates of $H$ and $K$ as well, we conclude that $\left\langle H^{x}, K^{y}\right\rangle=G$ for all $x, y \in G$.

Now, let $M$ be a maximal normal subgroup of $G$. Then $(M, M \cap H) \in \mathscr{C}$ and $(M, M \cap K) \in \mathscr{C}$ by injectivity, and hence induction on $M \geq M \cap H, M \cap K$ implies $\left(M, M \cap H \cap K^{m}\right) \in \mathscr{C}$ for some $m \in M$. By injectivity there exists some $S \leq G$ with $(G, S) \in \mathscr{C}$ and $S \cap M \leq H \cap K^{m}$. Note that $(M, S \cap M) \in \mathscr{C}$ with $S \cap M \leq H \cap M$. Hence, $(H \cap M, S \cap M) \in \mathscr{C}$. Now $H \cap M \unlhd H$, so applying injectivity, we can find a $T \leq H$ with $(H, T) \in \mathscr{C}$ and $T \cap M \leq S \cap M \leq H \cap M$. But, as we showed at the beginning of the proof, this can only happen if $T=H$. Hence $S \cap M=H \cap M$. We can apply an identical argument to $K^{m}$ to conclude that $S \cap M=K^{m} \cap M$ also. We have thus shown that $H \cap M=K^{m} \cap M$. Call this group $N$. Note that $H$ and $K^{m}$ both normalise $N$. Hence $G=\left\langle H, K^{m}\right\rangle \leq N_{G}(N)$, and we conclude that $N$ is normal in $G$.

Let $[G: M]=p$. Since $H$ and $K$ are not contained in $M$, we have $[H: N]=p$ and $[K: N]=p$. Let $P$ be a Sylow $p$-subgroup of $G$. Then $H^{x} \leq P N$ and $K^{y} \leq P N$ for some $x, y \in G$. But now $G=\left\langle H^{x}, K^{y}\right\rangle \leq P N$. Then $G / N$ is a $p$-group, hence nilpotent, and $N \leq H<G$. This would mean $H$ would have to be contained in a proper normal subgroup of $G$. This is the final contradiction that proves the result.

The problem of seeking other generalisations of Sylow's theorem then becomes one of seeking other conditions which, like injectivity and projectivity, will make a complete subgroup class conjective.

\section{The associated class}

If $\mathscr{C}$ is any subgroup class of the class $\mathscr{X}$, we can associate with it a subclass $\mathscr{C}^{\dagger}$ of $\mathscr{X}$ defined as follows

$$
\mathscr{C}^{\dagger}=\{G: G \in \mathscr{X},(G, H) \in \mathscr{C} \text { if and only if } H=G\}
$$

Proposition 7. If $\mathscr{X}$ is a Schunck class and $\mathscr{C}$ is a bounded projective subgroup class in $\mathscr{X}$, then the class $\mathscr{C}^{\dagger}$ formed above is also a Schunck class. 
PROOF. The proof is a straightforward verification of the Schunck class axioms.

If $K \unlhd G \in \mathscr{C}^{\dagger}$, then $G \in \mathscr{X}$, and so $G / K \in \mathscr{X}$ since $\mathscr{X}$ is a Schunck class. Let $(G / K, H / K) \in \mathscr{C}$. By projectivity $(G, H) \in \mathscr{C}$, and $H=G$ as $G \in \mathscr{C}^{\dagger}$. So $H / K=G / K$, and $G / K \in \mathscr{C}^{\dagger}$.

Suppose that every primitive quotient group of $G$ is in $\mathscr{C}^{\dagger}$. Then every primitive quotient group of $G$ is in $\mathscr{X}$, and as $\mathscr{X}$ is a Schunck class, $G \in \mathscr{X}$. To show $G \in \mathscr{C}^{\dagger}$ let $(G, H) \in \mathscr{C}$. By boundedness of $\mathscr{C}$ we can assume without loss of generality that $H$ is maximal. Let $K=\operatorname{Cor}_{G}(H)$, so $G / K$ is primitive, and is in $\mathscr{C}^{\dagger}$. By projectivity $(G / K, H / K) \in \mathscr{C}$ so $H / K=G / K$. But this implies $H=G$, and so $G \in \mathscr{C}^{\dagger}$ as claimed

PROPOSITION 8. If $\mathscr{X}$ is a Fitting class and $\mathscr{C}$ is a bounded injective subgroup class in $\mathscr{X}$, then the class $\mathscr{C}^{\dagger}$ is also a Fitting class.

PROOF. Once again, the proof is a straightforward verification of axioms.

Suppose $N \unlhd G \in \mathscr{C}^{\dagger}$. As $\mathscr{C}^{\dagger} \subset \mathscr{X}$, we have $G \in \mathscr{X}$ and hence $N \in \mathscr{X}$ as $\mathscr{X}$ is Fitting. Let $(N, K) \in \mathscr{C}$. By injectivity there exists $S \leq G$ with $(G, S) \in \mathscr{C}$ and $S \cap N \leq K$. As $G \in \mathscr{C}^{\dagger}$ we must have $S=G$. So $K=N$ and $N \in \mathscr{C}^{\dagger}$.

Suppose that $G=H_{1} H_{2}$ with $H_{i} \in \mathscr{C}^{\dagger}$ and $H_{i} \unlhd G$. Note that $H_{1}, H_{2} \in \mathscr{X}$, hence $G \in \mathscr{X}$ as $\mathscr{X}$ is Fitting.

Suppose that $(G, K) \in \mathscr{C}$. Now $\left(H_{1}, H_{1} \cap K\right) \in \mathscr{C}$ by injectivity. But $H_{1} \in \mathscr{C}^{\dagger}$ implies $H_{1} \cap K=H_{1}$ and $H_{1} \leq K$. Similarly $H_{2} \leq K$ and $G=H_{1} H_{2} \leq K$. So $K=G$ and $G \in \mathscr{C}^{\dagger}$.

Note that in both of these theorems, closure and transitivity were not required. Mere boundedness sufficed for the proof.

This gives us a quick simple method of passing from a subgroup class to the associated group class which takes projective subgroup classes to Schunck classes and injective classes to Fitting classes. We note however that this association is not 1-1. In particular as we have seen there are at least two different complete subgroup classes associated with the class of all nilpotent groups, namely the projective class and the injective class. So the map $\dagger$ is non-invertible. We can however find partial inverse maps to $\dagger$ by imposing extra conditions. Note that, if $\mathscr{K}$ is Schunck, then $\mathscr{P}(\mathscr{K})^{\dagger}=\mathscr{K}$. Similarly, if $\mathscr{K}$ is Fitting then $\mathscr{I}(\mathscr{K})^{\dagger}=\mathscr{K}$.

It is somewhat unsatisfying to have $\mathscr{P}(\mathscr{K})$ and $\mathscr{I}(\mathscr{K})$ defined in terms of projectors and injectors. In particular it prevents us from using Theorems 5 and 6 as alternative proofs of the existence and uniqueness up to conjugacy of injectors and projectors in Schunck and Fitting classes. The author has contrived an alternate definition of $\mathscr{P}(\mathscr{K})$ which does not use projectors. However the definition is a complicated one, and the work required to prove that it gives a complete projective subgroup class 
is considerable, rendering it of dubious utility. The situation with regard to $\mathscr{I}(\mathscr{K})$ appears similar.

\section{Interjective subgroup classes}

In this section we define a new property of subgroup classes which we shall choose to call interjectivity. We will then prove that interjective complete subgroup classes are conjective.

DEFINITION 9. If $G$ is a finite group, then we define an interval $(N \unlhd M)$ of $G$ to be a pair of nested normal subgroups of $G$.

If $\mathscr{C}$ is a subgroup class of $\mathscr{X}$, then we say that $\mathscr{C}$ covers the interval $(N \unlhd M)$ of $G$ if

(a) $(G, H) \in \mathscr{C}, M / N \in \mathscr{X}$ implies $(M / N,(N H \cap M) / N) \in \mathscr{C}$.

(b) $G \in \mathscr{X}$ and $(M / N, T / N) \in \mathscr{C}$ implies for every $R \leq G$ with $R \cap M \geq T$ $\exists S \leq R$ with $(R, S) \in \mathscr{C}$ and $S N \cap M \leq T$

If $\mathscr{C}$ is a subgroup class of $\mathscr{X}$ and $G \in \mathscr{X}$ then the cover set $\mathscr{C}_{G}$ of $G$ is the set of intervals of $G$ which are covered by $\mathscr{C}$.

The definition requires comment. The most natural definition would deal only with the case that $R==G$ in part (b). However this definition later proves to be too weak to complete the proofs of theorems like Theorem 11. It is also worth noting that the definition implies that all intervals $(N \unlhd M)$ for which $M / N \notin \mathscr{X}$ are covered by $\mathscr{C}$. While this may seem strange, intervals of this type do not arise in the kinds of situations where we shall use the definition. I merely point this out as a technical oddity.

Note that injectivity and projectivity of a subgroup class can both be expressed as conditions on cover sets. In particular a subgroup class $\mathscr{C}$ is projective if and only if $\mathscr{C}_{G}$ contains every interval of the form $(N \unlhd G)$, for each $G \in \mathscr{X}$. Similarly a subgroup class is injective if and only if $\mathscr{C}_{G}$ contains every interval of the form $(1 \unlhd M)$, for each $G \in \mathscr{X}$.

This encourages us to seek generalisations of injectivity and projectivity which take the form of conditions on cover sets. The condition below achieves this aim, albeit in a rather blunt fashion, by forming a simple hybrid of the injectivity and projectivity conditions. The author suspects that better (that is, more general, more elegant) conditions on cover sets exist. In particular the most general condition on cover sets which enables conjectivity to be proved for complete subgroup classes would be a worthy goal. 
DEFINITION 10. A subgroup class $\mathscr{C}$ of $\mathscr{X}$ is said to be interjective if, for each $G \in \mathscr{X}$, the cover set of $G$ contains either all intervals of the form $(N \unlhd G)$ where $N$ is a minimal normal subgroup of $G$, or all interval of the form $(1 \unlhd M)$ where $M$ is a maximal normal subgroup of $G$.

THEOREM 11. If $\mathscr{C}$ is a complete interjective subgroup class of the class of all solvable groups, then $\mathscr{C}$ is conjective. We call the $\mathscr{C}$-conjectors of an interjective class interjectors.

PROOF. Let $G$ be a group of minimal order in which the conjectivity condition fails. By interjectivity the cover set of $G$ either includes all intervals $(N \unlhd G)$ for $N$ minimal normal in $G$, or includes all intervals of the form $(1 \unlhd M)$ for $M$ maximal normal in $G$. In the first case the proof follows along similar lines to the proof of Theorem 5. In the second case the proof follows the method of Theorem 6.

Case 1: Assume that $\mathscr{C}_{G}$ contains $(N \unlhd G)$ for all minimal normal subgroups $N$. Choose $H, K \leq G$ for which conjectivity fails so that $[G: H]$ and $[G: K]$ are minimal.

By the argument in the proof of Theorem 5, both $H$ and $K$ are maximal in $G$. Assume that $H$ contains a minimal normal subgroup $N$.

If $N \not K$ then $N K=G$ with $N \cap K=1$ so $G / N \cong K$. Also $H=(H \cap K) N$ so that $H / N \cong(H \cap K)$. Since $\mathscr{C}$ covers $(N \unlhd G)$ we conclude $(G / N, H / N) \in \mathscr{C}$ and thus $(K, K \cap H) \in \mathscr{C}$. But transitivity now gives $(G, K \cap H) \in \mathscr{C}$ which is contradictory since $H$ and $K$ do not obey the conjectivity condition. So $N \leq K$. But now $(G / N, H / N) \in \mathscr{C}$ and $(G / N, K / N) \in \mathscr{C}$ since $\mathscr{C}$ covers $(N \unlhd G)$. But now conjectivity holds in $G / N$. Thus $\left(G / N,\left(H \cap K^{g}\right) / N\right) \in \mathscr{C}$ for some $g \in G$. But this implies there exists $(G, S) \in \mathscr{C}$ with $S \leq H \cap K^{g}$. By boundedness $\left(G, H \cap K^{g}\right) \in \mathscr{C}$, once again a contradiction.

Hence $H$ and $K$ are both stabilisers of $G$. Hence are conjugate, a final contradiction.

Case 2: Assume that $\mathscr{C}_{G}$ contains $(1 \unlhd M)$ for all maximal normal subgroups $M$. Choose $H, K \leq G$ for which conjectivity fails so that $|H|$ and $|K|$ are minimal.

By the argument in the proof of Theorem 6, if $(H, S) \in \mathscr{C}$ then $S=H$, with this also being true for $K$. The same arguments as in Theorem 6 also show that neither $H$ nor $K$ can be contained in a maximal normal subgroup of $G$, and that $\left\langle H^{x}, K^{y}\right\rangle=G$ for all $x, y \in G$.

Now, let $M$ be a maximal normal subgroup of $G$. Since $\mathscr{C}$ covers $(1 \unlhd M)$ we have $(M, M \cap H) \in \mathscr{C}$ and $(M, M \cap K) \in \mathscr{C}$. Thus $\left(M, M \cap H \cap K^{m}\right) \in \mathscr{C}$ for some $m \in M$.

Since $\mathscr{C}$ covers $(1 \unlhd M)$, we can apply the cover condition with $R=H$ to conclude that there exists $S \leq H$ with $(H, S) \in \mathscr{C}$ and $S \cap M \leq H \cap K^{m} \cap M$. But $(H, S) \in \mathscr{C}$ implies $S=H$, and hence $H \cap M \leq K^{m} \cap M$. Similarly we can apply the cover condition with $R=K^{m}$ to conclude that $K^{m} \cap M \leq H \cap M$. Hence these two 
groups are equal, and we can write $T=H \cap M=K^{m} \cap M$. Since $T$ is normalised by both $H$ and $K^{m}, T \unlhd G$.

Let $[G: M]=p$. Since $H$ and $K$ are not contained in $M$, we have $[H: T]=p$ and $[K: T]=p$. Let $P$ be a Sylow $p$-subgroup of $G$. Conjugate $H$ and $K$ so that $H^{x} \cap P$ is a Sylow subgroup of $H^{x}$ and $K^{y} \cap P$ is a Sylow subgroup of $K^{y}$. Then $G=\left\langle H^{x}, K^{y}\right\rangle \leq P T$. But now $G / T$ is nilpotent, and $T \leq H<G$. So $H$ would have to be contained in a proper normal subgroup of $G$, a contradiction.

Much more remains to be done. There are new classes to be defined and explored, and most pressingly new examples that need building. We will content ourselves merely with noting that injectors and projectors may not be the end of the story, and that subgroup classes appear a most promising framework for developing still further generalisations of Sylow's theorem, surely the most fruitful theorem in group theory.

\section{References}

[1] Klaus Doerk and Trevor Hawkes, 'Finite soluble groups', in: Expositions in mathematics A (De Gruyter, Berlin, 1992).

Department of Mathematics

University of Waikato

Private Bag 3105

Hamilton

New Zealand

e-mail: hawthorn@waikato.ac.nz 